\title{
3D Viscoelastic Anisotropic Seismic Modeling with High-Order Mimetic Finite Differences
}

\author{
Miguel Ferrer, Josep de la Puente, Albert Farrés, and José E. Castillo
}

\begin{abstract}
We present a scheme to solve three-dimensional viscoelastic anisotropic wave propagation on structured staggered grids. The scheme uses a fully-staggered grid (FSG) or Lebedev grid (Lebedev, J Sov Comput Math Math Phys 4:449 465, 1964; Rubio et al. Comput Geosci 70:181-189, 2014), which allows for arbitrary anisotropy as well as grid deformation. This is useful when attempting to incorporate a bathymetry or topography in the model. The correct representation of surface waves is achieved by means of using high-order mimetic operators (Castillo and Grone, SIAM J Matrix Anal Appl 25:128-142, 2003; Castillo and Miranda, Mimetic discretization methods. CRC Press, Boca Raton, 2013), which allow for an accurate, compact and spatially high-order solution at the physical boundary condition. Furthermore, viscoelastic attenuation is represented with a generalized Maxwell body approximation, which requires of auxiliary variables to model the convolutional behavior of the stresses in lossy media. We present the scheme's accuracy with a series of tests against analytical and numerical solutions. Similarly we show the scheme's performance in high-performance computing platforms. Due to its accuracy and simple pre- and post-processing, the scheme is attractive for carrying out thousands of simulations in quick succession, as is necessary in many geophysical forward and inverse problems both for the industry and academia.
\end{abstract}

\section{Introduction}

Seismic waves occur when the subsurface is excited, by an internal event (e.g. an earthquake, an underground explosion) or an external event (e.g. the impact of a meteorite, a landslide). The behaviour of such waves can be described by means of a

M. Ferrer $(\triangle) \bullet$ J. de la Puente $\bullet$ A. Farrés

Computer Applications in Science and Engineering, Barcelona Supercomputing Center,

Jordi Girona 29, 08034 Barcelona, Spain

e-mail: miguel.ferrer@bsc.es; josep.delapuente@bsc.es; albert.farres@bsc.es

J.E. Castillo

Computational Science Research Center, San Diego State University, 5500 Campanile Drive,

San Diego, CA 92182-7720, USA

e-mail: jcastillo@mail.sdsu.edu

(C) Springer International Publishing Switzerland 2015

R.M. Kirby et al. (eds.), Spectral and High Order Methods for Partial Differential

Equations ICOSAHOM 2014, Lecture Notes in Computational Science

and Engineering 106, DOI 10.1007/978-3-319-19800-2_18 
hierarchy of physical laws that represent ever more accurately observed phenomena. For certain applications, waves can be represented as rays, although some wave phenomena require of mechanical laws that properly describe their properties. At medium to long scales, seismic waves can be fully described with an anisotropic viscoelastic theory. Anisotropy describes the properties of some solids to support waves moving with different speeds when they travel in different directions. In rocks, anisotropy can be due to intrinsic crystalline properties or a macroscopic representation of fine sediment layering. Viscoelasticity is a macroscopic property which accounts for energy losses observed in the subsurface. When having good models of the subsurface properties, anisotropic viscoelastic modelling allows us to obtain synthetic seismic waves which behave very similarly to observed waves. A very popular approach for modelling seismic waves is the staggeredgrid time-domain finite-difference method $[14,16,20,21]$. This method is very efficient for large simulations. However, it presents limitations when modelling strong anisotropy [9] or topography [8]. An improvement to the method is the fullystaggered grid (FSG) method $[13,15]$ which naturally supports arbitrary anisotropy. More recently [4] showed that the method can be further modified by using mimetic operators and deformed grids to model topography with high precision. In this paper we show how the mimetic FSG finite-difference method can be improved by adding support for viscoelastic materials with Generalized Maxwell Body (GMB, see [5]) rheology.

\section{Viscoelastic Wave Propagation}

Viscoelastic waves, in time-domain velocity-stress formulation, are governed by the PDE

$$
\begin{gathered}
\frac{\partial \mathbf{S}}{\partial t}=\mathbf{C} * \mathbf{E}, \\
\frac{\partial \mathbf{v}}{\partial t}=\frac{1}{\rho} \mathbf{T}
\end{gathered}
$$

where the stress tensor in vector form is $\mathbf{S}=\left(\sigma_{x x}, \sigma_{y y}, \sigma_{z z}, \sigma_{y z}, \sigma_{x z}, \sigma_{x y}\right)^{T}$, the strainrate tensor in vector form is $\mathbf{E}=\left(\dot{\varepsilon}_{x x}, \dot{\varepsilon}_{y y}, \dot{\varepsilon}_{z z}, \dot{\varepsilon}_{y z}, \dot{\varepsilon}_{x z}, \dot{\varepsilon}_{x y}\right)^{T}, \mathbf{C}$ is the stiffness matrix, $\mathbf{v}$ is the particle velocity vector, $\rho$ the density and $\mathbf{T} \equiv \partial \sigma_{i j} / \partial x_{j}$ which is related to the gradients of the tractions in planes perpendicular to all three Cartesian directions $x, y$ and $z$. Equation (1) is sufficient to describe waves propagating through a solid lossy material. In general, both compressional $P$ and shear $S$ wave modes are supported in viscoelastic media. The convolution in the equation becomes a normal product in case the medium is lossless (e.g. elastic). Viscoelastic effects are generally accounted for with quality factors $Q_{P}$ and $Q_{S}$ which are lower the more attenuated the wave mode is and, additionally, are reported to be almost frequency independent. Many mechanical models exist to represent accurately viscoelastic 
effects in geophysics, although one of the most accurate is the Generalized Maxwell Body (GMB) rheology (see [17] for a complete overview). When using GMB, we can rewrite (1) as

$$
\begin{gathered}
\frac{\partial \mathbf{S}}{\partial t}=\tilde{\mathbf{C}} \mathbf{E}-\sum_{l=1}^{n} \mathbf{Y}^{l} \mathbf{A}^{l}, \\
\frac{\partial \mathbf{v}}{\partial t}=\frac{1}{\rho} \mathbf{T}, \\
\frac{\partial \mathbf{A}^{l}}{\partial t}=\omega_{l}\left(\mathbf{A}^{l}-\mathbf{E}\right),
\end{gathered}
$$

where $n$ denotes the number of Maxwell mechanisms used, $\mathbf{Y}^{l}$ are viscoelastic coefficient matrices, $\mathbf{A}^{l}=\left(a_{x x}, a_{y y}, a_{z z}, a_{y z}, a_{x z}, a_{x y}\right)^{T}$ are the anelastic variables related to the strain rates and $\omega_{l}$ is the characteristic frequency of each mechanism. Notice that the stiffness matrix in (2) refers to the unrelaxed stiffness $\tilde{\mathbf{C}}$, which refers to the value of $\mathbf{C}$ at very high frequencies. Equation (2) allows us to model viscoelastic waves without convolution operators, which have been substituted by extra (anelastic) variables in our system. This makes the simulation of viscoelastic waves affordable in the time domain. Explicitly, we have

$$
\tilde{\mathbf{C}}=\left(\begin{array}{llllll}
\tilde{c}_{11} & \tilde{c}_{12} & \tilde{c}_{13} & c_{14} & c_{15} & c_{16} \\
\tilde{c}_{12} & \tilde{c}_{22} & \tilde{c}_{23} & c_{24} & c_{25} & c_{26} \\
\tilde{c}_{13} & \tilde{c}_{23} & \tilde{c}_{33} & c_{34} & c_{35} & c_{36} \\
c_{14} & c_{24} & c_{34} & \tilde{c}_{44} & c_{45} & c_{46} \\
c_{15} & c_{25} & c_{35} & c_{45} & \tilde{c}_{55} & c_{56} \\
c_{16} & c_{26} & c_{36} & c_{46} & c_{56} & \tilde{c}_{66}
\end{array}\right), \mathbf{Y}^{l}=\left(\begin{array}{cccccc}
Y_{l}^{P} & Y_{l}^{\lambda} & Y_{l}^{\lambda} & 0 & 0 & 0 \\
Y_{l}^{\lambda} & Y_{l}^{P} & Y_{l}^{\lambda} & 0 & 0 & 0 \\
Y_{l}^{\lambda} & Y_{l}^{\lambda} & Y_{l}^{P} & 0 & 0 & 0 \\
0 & 0 & 0 & Y_{l}^{S} & 0 & 0 \\
0 & 0 & 0 & 0 & Y_{l}^{S} & 0 \\
0 & 0 & 0 & 0 & 0 & Y_{l}^{S}
\end{array}\right)
$$

so that the material can be anisotropic but we only accept isotropy in the attenuative properties of the material. The $\omega_{l}$ values are chosen to cover our desired bandwidth evenly in the logarithmic scale. Then, the coefficients in $\mathbf{Y}^{l}$ can be found, if we use auxiliary halfway $\omega_{k}$ points with $k=1, \cdots, 2 n-1$, by using

$$
\begin{aligned}
& Q_{v}^{-1}\left(\omega_{k}\right)=\sum_{l=1}^{n} \frac{\omega_{k} \omega_{l}+\omega_{l}^{2} Q_{v}^{-1}\left(\omega_{k}\right)}{\omega_{l}^{2}+\omega_{k}^{2}} Y_{l}^{v} \text { with } v=P, S . \\
& Y_{l}^{\lambda}=\frac{P}{L} Y_{l}^{P}-\frac{2 S}{L} Y_{l}^{S}
\end{aligned}
$$

where $Q_{P}$ and $Q_{S}$ are locally constant values and $P=c_{i i} / 3$ with $i=1,2,3$, $S=c_{i i} / 3$ with $i=4,5,6$ and $L=P-2 S$. Finally, the unrelaxed stiffness components must be found so that the input velocities match as well as possible 
the phase velocity at our peak frequency $\omega_{0}$. This can be achieved using

$$
\begin{aligned}
& \Theta_{1}^{v}=1-\sum_{l=1}^{n} Y_{l}^{v} \frac{1}{1+\left(\omega_{0} / \omega_{l}\right)^{2}}, \Theta_{2}^{v}=\sum_{l=1}^{n} Y_{l}^{v} \frac{\omega_{0} / \omega_{l}}{1+\left(\omega_{0} / \omega_{l}\right)^{2}}, \\
& U^{v}=\frac{\sqrt{\left(\Theta_{1}^{v}\right)^{2}+\left(\Theta_{2}^{v}\right)^{2}}+\Theta_{1}^{v}}{2\left(\Theta_{1}^{v}\right)^{2}+2\left(\Theta_{2}^{v}\right)^{2}}, \quad \text { with } v=P, S
\end{aligned}
$$

The values $U^{P}$ and $U^{S}$ can be used to obtain the unrelaxed stiffness values following

$$
\begin{array}{ll}
\tilde{c}_{i i}=c_{i i} U^{P}, & \text { for } i=1,2,3, \\
\tilde{c}_{i i}=c_{i i} U^{S}, & \text { for } i=4,5,6, \\
\tilde{c}_{i j}=c_{i j} \frac{P U^{P}-2 U^{S}}{L}, & \text { for } i, j=1,2,3 \text { and } i \neq j .
\end{array}
$$

After initializing the parameters with Eqs. (3)-(6), we solve system (2) using an FSG finite-difference method, where all spatial derivatives are substituted by mimetic operators [2-4, 18]. In addition, we use a leap-frog explicit scheme for the time integration which benefits from the corrections in [11] for reducing storage in this configuration. Notice that in time-domain explicit seismic modelling applications, time integration beyond second order is rare, as errors in the form of dispersion are dominated by the spatial discretization at the relevant frequency and propagation distances [6].

\section{2D Homogeneous Test}

First of all we wish to verify the accuracy of our scheme when handling elastic and viscoelastic wave propagation. To that goal we set up a simple $2 \mathrm{D}$ test in homogenous material for which an analytical solution exists [1]. We use a material with $v_{P}=6000 \mathrm{~m} / \mathrm{s}, v_{S}=3464 \mathrm{~m} / \mathrm{s}$ and $\rho=2700 \mathrm{~kg} / \mathrm{m}^{3}$. We then set $Q_{P}=60$ and $Q_{S}=30$ for the viscoelastic case and $Q_{P}=Q_{S}=\infty$ for the elastic case. The source is a force acting with a Ricker wavelet having its energy peak at $10 \mathrm{~Hz}$. A receiver is placed $1500 \mathrm{~m}$ away from the source along the source direction. Viscoelasticity is modelled with $n=3$ using a bandwidth of $100 \mathrm{~Hz}$ centered on the source's peak frequency. The model was discretized with a $201 \times 201$ grid of spacing $30 \mathrm{~m}$. In Fig. 1 left, we plot the wave velocities depending on the frequency as a consequence of the GMB mechanisms. Similarly we can observe how the desired $Q$ value is fitted along our bandwidth. In Fig. 1 right, we show the fit between analytical and numerical solutions for both the elastic and viscoelastic cases. We observe no quality degradation due to using viscoelasticity in our algorithm. Furthermore, we can observe how the P-wave (earlier) and S-wave (later) arrivals are both damped, being the energy loss stronger for the $\mathrm{S}$-wave due to the lower (i.e. more attenuating) $Q$ 

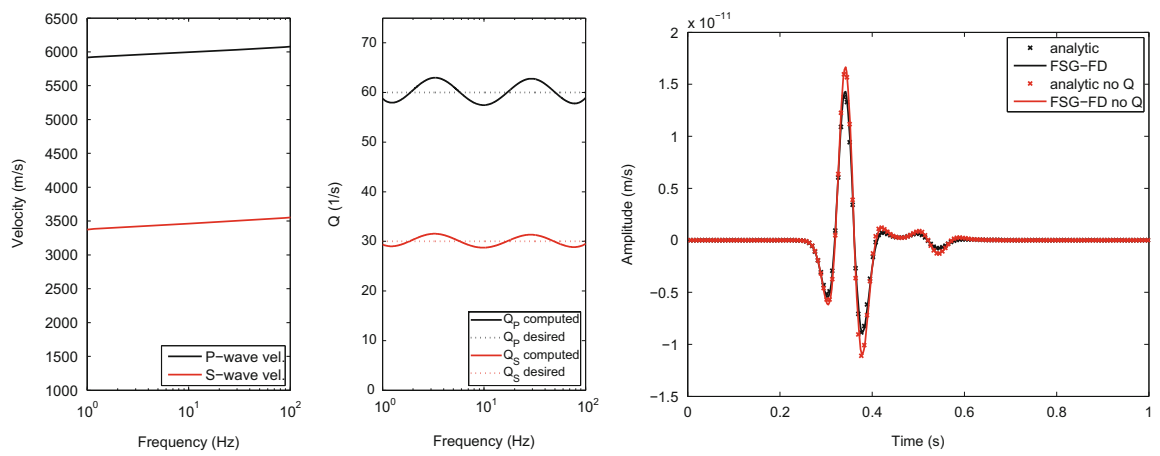

Fig. 1 Left: velocity dispersion and attenuation fit for both P- and S-wave. Right: simulation and analytical solutions for elastic and viscoelastic case

value for this wave mode. Similarly, we can observe that our coefficient computation algorithm enabled a nearly zero phase difference between the elastic and viscoelastic runs, as is expected from our waves concentrating energy around the peak of the wavelet.

\section{3D Heterogeneous Test}

We have built a large 3D elastic and its equivalent viscoelastic model. The model is cubic and composed of 27 small subcubes, each of them with different physical properties. The model is challenging because it displays very large contrasts in the material properties. P-wave velocities range from 1000 to $5000 \mathrm{~m} / \mathrm{s}$ and Poisson ratios from values of $0.2-0.45$, which results in $\mathrm{S}$-wave velocities ranging from 408 to $3535 \mathrm{~m} / \mathrm{s}$. Densities range from 1200 to $2700 \mathrm{~kg} / \mathrm{m}^{3}$. In the viscoelastic case, $Q_{P}$ takes values from 50 to 250 and $Q_{S}$ from 20 to 176 . We have an explosive source located at the middle of the domain with a Ricker wavelet having peak frequency at $20 \mathrm{~Hz}$. A total of six receivers are located at the center of each of the domain's quadrilateral faces. The time sampling is $\Delta t=0.00016 \mathrm{~s}$ and the spatial sampling is equal in all directions to $2.5 \mathrm{~m}$. The volume is composed of $501 \times 501 \times 501$ cells and the simulation lasts for 10,000 iterations. CPML [10] boundary conditions are set everywhere. The results of the simulation with and without viscous mechanisms can be seen in Fig. 2. We observe that the strong heterogeneity generates many wave arrivals. The viscoelastic simulation is mostly in-phase with the elastic one, displaying different degrees of energy loss depending on the actual arrival and receiver. We conclude that the method is robust in strongly heterogeneous cases, including attenuation in $3 \mathrm{D}$, when using a wide range of realistic values for the material properties. For large scenarios like this, we employ a hybrid parallel approach OpenMP/MPI for distributed memory computer clusters. 

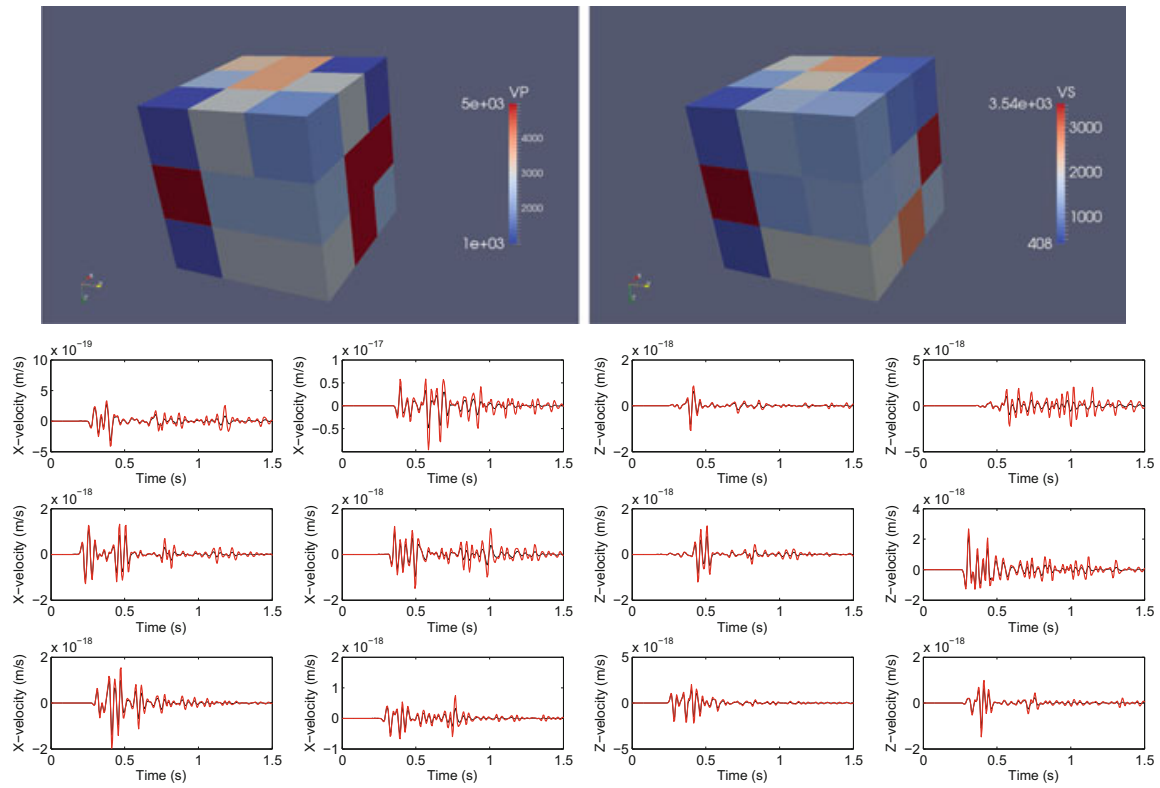

Fig. 2 Description of model for P-wave velocity and S-wave velocity (top) and seismograms recorded at all six locations, for the $x$ and $z$ components of the particle velocity vector (bottom)
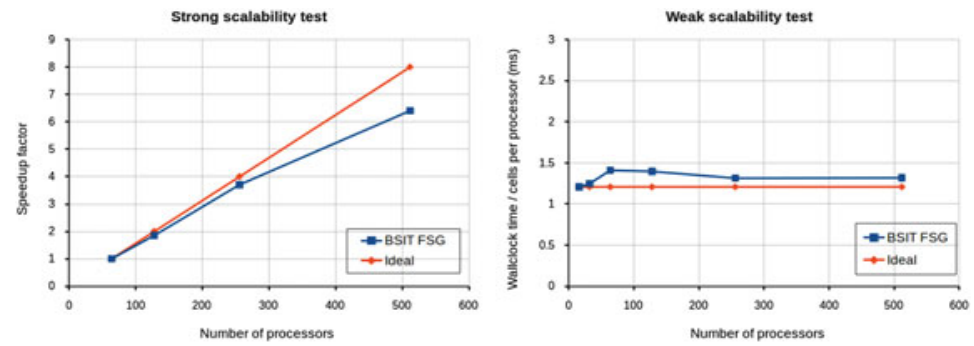

Fig. 3 Strong and weak scalability tests performed on the MareNostrum supercomputer at the Barcelona Supercomputing Center

The simulation code has been developed with BSIT [7, 19] achieving 69 GFLOPS per Intel E5-2670 16-core node. A scalability test is provided in Fig. 3.

\section{Discussion}

As a final check for the correctness of our results we can quantify the dispersion and amplitude differences for our two examples. We perform a time-frequency analysis of the elastic and viscoelastic solutions using the definitions of Kristekova 

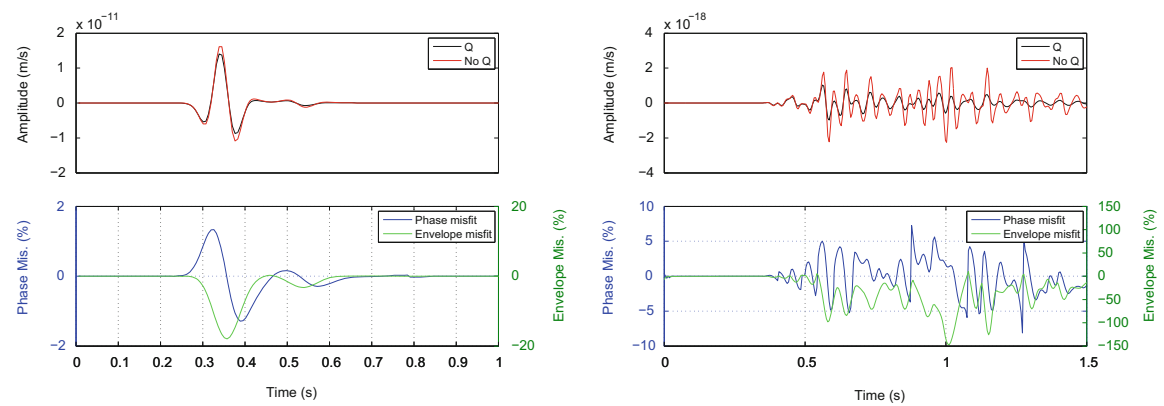

Fig. 4 Phase and envelope misfits for the homogeneous case and for a randomly chosen receiver in the $3 \mathrm{D}$ heterogeneous case

et al. [12]. This allows us to completely separate phase misfits from envelope misfits. By looking at the misfits for the homogeneous case, in Fig. 4 left, we can see that the envelope misfits are negative everywhere, which corresponds to the observed (and expected) amplitude loss in the viscoelastic case. More interesting is the phase misfit. We observe that, for both the $\mathrm{P}$ and $\mathrm{S}$ arrivals, we have first a slight phase misfit increase which is followed by a slight misfit decrease after the wave peak. This indicates that each wave arrival is being dispersively separated into faster wave components and slower wave components. This is what we expect, and corresponds to the dispersion curves in Fig. 1 where higher frequency modes travel faster than lower frequency modes. We remark again that this dispersive behaviour is expected in physically sound viscoelastic rheologies [1]. Furthermore, the phase misfit tends to average out along during each arrival, indicating that the central frequencies travel at the correct velocity. In Fig. 4 right we have a more complex scenario, but nevertheless displaying the same behaviour: phase misfits increase and then decrease for an overall in-phase propagation although with signs of dispersion. The envelope misfit, however is always negative and quite large. Notice that as waves have travelled more cycles, the effects of dispersion and attenuation are also stronger.

\section{Conclusions}

We have described the upgrade of the FSG time-domain mimetic finite-difference method to support viscoelastic attenuation accurately. Our approach is based on a Generalized Maxwell Body mechanism which allows us to correctly model the dispersive behaviour of viscoelastic waves. We make an effort in finding ways to obtain attenuating parameters that respect our wave velocity at the center of our frequency bandwidth and are quasi-flat throughout it. The resulting algorithm has been tested against an analytical solution obtaining an excellent agreement with it. 
Furthermore, we have shown that the method is robust enough to tackle cases of extreme heterogeneity in large 3D scenarios.

Acknowledgements The authors want to thank Repsol for the permission to publish the present research, carried out at the Repsol-BSC Research Center as a part of the Kaleidoscope Project. This project has received funding from the European Union's Horizon 2020 research and innovation programme under the Marie Sklodowska-Curie grant agreement No 644602.

\section{References}

1. J. Carcione, Wave Fields in Real Media: Wave Propagation in Anisotropic, Anelastic and Porous Media. Handbook of Geophysical Exploration, Seismic Exploration (Pergamon Press, Oxford, 2002)

2. J. Castillo, R. Grone, A matrix analysis approach to higher-order approximations for divergence and gradients satisfying a global conservation law. SIAM J. Matrix Anal. Appl. 25, 128-142 (2003)

3. J.E. Castillo, G.F. Miranda, Mimetic Discretization Methods (CRC Press, Boca Raton, 2013)

4. J. de la Puente, M. Ferrer, M. Hanzich, J.E. Castillo, J.M. Cela, Effects of free-surface topography on moving-seismic-source modeling. Geophysics 79(3), T125-T141 (2014)

5. H. Emmerich, M. Korn, Incorporation of attenuation into time-domain computations of seismic wave fields. Geophysics 52, 1252-1264 (1987)

6. A. Fichtner, Full Seismic Waveform Modelling and Inversion (Springer, Heidelberg, 2010)

7. M. Hanzich, J. Rodriguez, N. Gutierrez, J. de la Puente, J. Cela, Using HPC software frameworks for developing BSIT: a geophysical imaging tool. In: Proceedings of WCCM XI, ECCM V, ECFD VI, vol. III (2014), pp. 2019-2030

8. S. Hestholm, Three-dimensional finite difference viscoelastic wave modelling including surface topography. Geophys. J. Int. 139(3), 852-878 (1999)

9. H. Igel, P. Mora, B. Riollet, Anisotropic wave propagation through finite-difference grids. Geophysics 60, 1203-1216 (1995)

10. D. Komatitsch, R. Martin, An unsplit convolutional perfectly matched layer improved at grazing incidence for the seismic wave equation. Geophysics 72(5), SM155-SM167 (2007)

11. J. Kristek, P. Moczo, Seismic-wave propagation in viscoelastic media with material discontinuities: a 3D fourth-order staggered-grid finite-difference modeling. Bull. Seismol. Soc. Am. 93, 2273-2280 (2003)

12. M. Kristeková, J. Kristek, P. Moczo, S. Day, Misfit criteria for quantitative comparison of seismograms. Bull. Seismol. Soc. Am. 96(5), 1836-1850 (2006)

13. V. Lebedev, Difference analogies of orthogonal decompositions of basic differential operators and some boundary value problems. J. Sov. Comput. Math. Math. Phys. 4, 449-465 (1964)

14. A.R. Levander, Fourth-order finite difference P-SV seismograms. Geophysics 53, 1425-1436 (1988)

15. V. Lisitsa, D. Vishnevskiy, Lebedev scheme for the numerical simulation of wave propagation in 3D anisotropic elasticity. Geophys. Prospect. 58(4), 619-635 (2010)

16. R. Madariaga, Dynamics of an expanding circular fault. Bull. Seismol. Soc. Am. 65, 163-182 (1976)

17. P. Moczo, J. Kristek, M. Galis, P. Pazak, M. Balazovjech, The finite-difference and finiteelement modeling of seismic wave propagation and earthquake motion. Acta Phys. Slovaca 57(2), 177-406 (2007)

18. O. Rojas, S. Day, J. Castillo, L.A. Dalguer, Modelling of rupture propagation using high-order mimetic finite differences. Geophys. J. Int. 172(2), 631-650 (2008) 
19. F. Rubio, M. Hanzich, A. Farrés, J. de la Puente, J. María Cela, Finite-difference staggered grids in GPUs for anisotropic elastic wave propagation simulation. Comput. Geosci. 70, 181189 (2014)

20. J. Virieux, SH-wave propagation in heterogeneous media: velocity-stress finite-difference method. Geophysics 49, 1933-1942 (1984)

21. J. Virieux, P-SV wave propagation in heterogeneous media: velocity-stress finite-difference method. Geophysics 51, 889-901 (1986) 